Kommentar: Auch wenn es sich hier um eine retrospektive Studie handelt, so hilft sie, diejenigen Kinder frühzeitig zu identifizieren, bei denen CPAP-Atemhilfe allein nicht ausreicht. Speziell männliche Frühgeborene unter $800 \mathrm{~g}$, die nach einer Stunde noch mehr als $25 \%$ Sauerstoff benötigen, um eine Sättigung von $86-94 \%$ zu erreichen, sollten eher großzügiger intubiert und mit Surfactant versorgt werden. Bei ihnen ist ein Scheitern der reinen CPAP-Atemhilfe wahrscheinlich. Eine Surfactantgabe vor der zweiten Lebensstunde kann zudem nach weiteren aktuellen Studien dazu beitragen, die Inzidenz der BPD und die Dauer der mechanischen Ventilation zu reduzieren.

Dr. Martin Claßen

\title{
Perianale streptogene Dermatitis: Penicillin oder Cephalosporin?
}

\section{Nach einer aktuellen Studie kann eine Cephalosporin-Therapie die Rezidiv- rate bei der perianalen streptogenen Dermatitis im Vergleich zur Behandlung mit Penicillin signifikant reduzieren.}

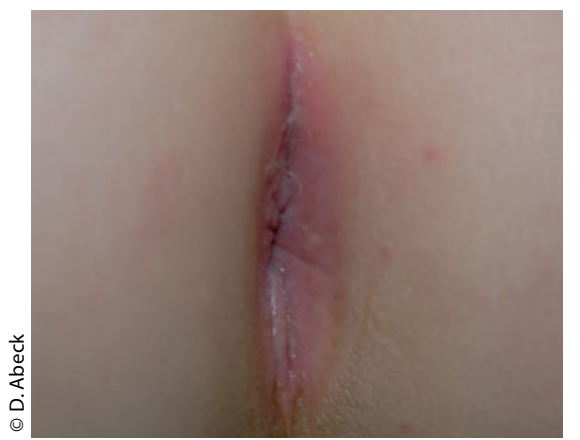

Perianale streptogene Dermatitis: scharf begrenztes Erythem im Bereich der Analfalte mit fibrinösen Auflagerungen

D e perianale streptogene Dermatitis (PSD) ist klinisch durch ein scharf begrenztes, nur gering schuppendes Erythem unterschiedlicher Intensität in der Analfalte gekennzeichnet. Der klinische Verdacht wird durch den Nachweis von $\beta$-hämolysierenden Streptokokken mittels Schnelltest oder Kultur bestätigt.

Eine orale Antibiotikabehandlung ist die Therapie der Wahl. Jedoch auch nach einer erfolgreichen Antibiotikabehandlung ist die Rezidivrate hoch.

In einer 81 Kinder mit PSD umfassenden Studie, die klinikeigene retrospektiv erhobene Daten wie auch Daten einer Metaanalyse umfasste, wurden die Beeinflussung der Rezidivrate unter Behandlung mit Penicillin bzw. Amoxicillin im Vergleich zur Behandlung mit einem $\beta$-Laktamase-stabilen Antibiotikum (überwiegend Cephalosporine) untersucht [1].
Die Untersuchung belegte die aus der Literatur bekannte hohe Rezidivquote, die bei $32,1 \% \operatorname{lag}(26 / 81)$, wobei $69,2 \%$ der Kinder (18/26) innerhalb der ersten sechs Wochen nach Behandlung ein PDS-Rezidiv entwickelten. Jedoch unterschied sich die Rezidivquote in Abhängigkeit vom verwendeten Antibiotikum signifikant: einer Rezidivrate von $38,1 \%$ (16/42) unter der Penicillin/Amoxicillin-Behandlung stand eine Rezidivrate von $27,8 \%$ (10/36) unter der Behandlung mit einem $\beta$-Laktamasestabilen Antibiotikum gegenüber (angepasste Odds Ratio: 2,02).

\section{Olson D, Edmondson MB. Outcomes in children treated for perineal group $A$ beta-hemolytic streptococcal dermatitis. Pediatr Infect Dis 2011; 30: 933-6 \\ 2. Meury SN et al. Randomized, compara- tive efficacy trial of oral penicicillin versus cefuroxime for perianal streptococcal dermatitis in children. J Pediatr 2008; 153: 799-802}

Kommentar: Diese Arbeit bestätigt die Ergebnisse einer früheren Untersuchung zur Therapie der PSD, in der Cefuroxim der klassischen Penicillin-Behandlung hinsichtlich dem klinischem Ansprechen und der Erregereradikation signifikant überlegen war [2]. Die Autoren der vorliegenden Studie diskutieren als Grund für die bessere Wirksamkeit der Cefuroxim-Therapie, dass durch die Verwendung eines $\beta$-Laktamase stabilen Antibiotikums auch weitere Erreger im Analbereich eliminiert werden, die sonst eine Wirksamkeit von Penicillin reduziert hätten.

Prof. Dr. Dietrich Abeck

\section{Crohn: Sind Antibiotika schuld?}

Bei Kindern, die häufiger mit Antibiotika behandelt werden, ist das Risiko erhöht, einen Morbus Crohn zu entwickeln. Ein Zusammenhang mit der Entstehung einer Colitis ulcerosa lässt sich dagegen nicht erkennen. Das ist das Ergebnis einer finnischen Registerstudie, in der 595 zwischen 1994 und 2008 geborene Kinder bis zu ihrem 16. Lebensjahr eine CED-Diagnose erhalten hatten. Ihr Antibiotikaverbrauch wurde mit dem von 2.380 gesunden Kindern verglichen. Eine solche Studie lässt sich in Finnland besonders gut durchführen, weil dort alle Antibiotikaverordnungen in einem zentralen Register erfasst werden.

Kinder mit mindestens sieben Antibiotikarezepten erkrankten doppelt so häufig wie die Kinder, für die zwischen null und sechs Rezepte ausgestellt worden waren. Besonders deutlich war der Zusammenhang zwischen Antibiotikagebrauch und M. Crohn bei Jungen und bei Kindern mit einer Diagnose vor dem elften Lebensjahr. Die Substanzklasse mit der höchsten Krankheitsinzidenz waren Cephalosporine. Dr. Beate Schumacher

Virta L et al. Am J Epidemiol 2012 Feb 24 [Epub ahead of print]

\section{Bei Koliken Allergene reduzieren}

Gegen die Säuglingskolik gibt es viele Geheimtipps. Australische Wissenschaftler haben die Literatur systematisch durchforstet, um herauszufinden, welche Behandlung am zuverlässigsten wieder Ruhe in Babybäuchen schafft. Dabei zeigten sich die besten Erfolge mit einer hypoallergenen Ernährung. In verschiedenen Studien verringerten sich die Koliken und damit die Schreidauer überwiegend dann, wenn hypoallerge Babynahrung, z. B. als Kasein- oder evtl. auch als Molke-Hydrolysate, gegeben wurden. Bei gestillten Kindern reduzierte eine allergenarme Ernährung der Mutter über eine Woche die Bauchschmerzen des Säuglings. Ballaststoffreiche Milchnahrung sowie die Gabe von Laktase hingegen zeigten keine Wirkung. Zwar ließen sich manche Babys mit Musik ablenken, insgesamt waren die Studien zu möglichen Verhaltensinterventionen wie etwa auch vermehrtem Herumtragen aber uneinheitlich und wenig aussagekräftig. Dr. Christine Starostzik

Hall B. et al. J Paediatr Child Health 2012; 48: 128-37 